\title{
The Fractional Residual Power Series Method for Solving a System of Linear Fractional Fredholm Integro-differential Equations
}

\author{
Prapart Pue-on \\ Mathematics and Applied Mathematics Research Unit, Department of Mathematics, Faculty of Science, \\ Mahasarakham University, Maha Sarakham 44150, Thailand
}

Received June 8, 2021; Revised September 16, 2021; Accepted September 28, 2021

Cite This Paper in the following Citation Styles

(a): [1] Prapart Pue-on, "The Fractional Residual Power Series Method for Solving a System of Linear Fractional Fredholm Integro-differential Equations," Mathematics and Statistics, Vol.9, No.5, pp. 792-798, 2021. DOI: 10.13189/ms.2021.090519

(b): Prapart Pue-on, (2021). The Fractional Residual Power Series Method for Solving a System of Linear Fractional Fredholm Integro-differential Equations. Mathematics and Statistics, 9(5), 792-798. DOI: 10.13189/ms.2021.090519

Copyright $@ 2021$ by authors, all rights reserved. Authors agree that this article remains permanently open access under the terms of the Creative Commons Attribution License 4.0 International License

\begin{abstract}
In this manuscript, the fractional residual power series (FRPS) method is employed in solving a system of linear fractional Fredholm integro-differential equations. The significant role of this system in various fields has attracted the attention of researchers for a decade. The definition of fractional derivative here is described in the Caputo sense. The proposed method relies on the generalized Taylor series expansion as well as the fact that the fractional derivative of stationary is zero. The process starts by constructing a residual function by supposing the finite order of an approximate power series solution that prescribes the initial conditions. Then, utilizing some conditions, the residual functions are converted to a linear system for the power series coefficients. Solving the linear system reveals the coefficients of the fractional power series solution. Finally, by substituting these coefficients into the supposed form of a solution, the approximate fractional power series solutions are derived. This technique has the advantage of being able to be applied directly to the problem and spending less time on computation. It is not only an easy method for implementation of the problem, but also provides productive results after a few iterations. Some problems with known solutions emphasize the procedure's simplicity and reliability. Moreover, the obtained exact solution demonstrated the efficiency and accuracy of the presented method.
\end{abstract}

Keywords Fractional Fredholm Integro-differential Equations, Caputo Fractional Derivatives, Residual Power Series Method, Exact Solution

\section{Introduction}

Fractional calculus came into existence from a question posed by L'Hôpital in a message to Leibniz in 1695 [20]. The essential question is about what a derivative of order $1 / 2$ is. Since then, an investigation of scholars has been started and fractional derivatives have been defined in many aspects by several mathematicians such as Riemann-Liouville, Caputo, Grunwald-Letnikov, Hadamard. These developments lead to the widespread use of fractional calculus in various areas.

In recent years, a fractional integro-differential equation system, one of the fractional calculus applications, has become a trending topic for investigation as it has been used as a mathematical model for a variety of phenomena. Unfortunately, solving this sort of system and related topics is extremely difficult and challenging because most of them do not have a precise solution. Many strategies have been devised to help estimate the solution to defeating the system, such as the Adomian decomposition method (ADM)[19], Homotopy analysis [26], B-spin method [2], Sadik decomposition method [21], Genocchi polynomial method [18], Chebyshev spectral method [25], Chebyshev pseudo-spectral method[11], Chebyshev wavelet method [12], approximation method based on Taylor expansion [5] and reference therein.

The system of linear fractional Fredholm integro-differential equations is one of the most interesting integro-differential systems that has attracted attention from many academics. This type of system is crucial in the fields of research and engineering. Some techniques, such as ADM [23] and Homotopy Perturbation Method (HPM) [22], have been employed to 
solve the system. Even if these approximation approaches are achieved, some restriction is required. As a result, researchers have been eager to find more practical, less constrained methods of searching for solutions.

The fractional residual power series (FRPS) method is a semi-analytic, powerful procedure based on the generalized Taylor series and a residual error function. Because no linearization, discretization, or perturbation is required, this method is efficient in addressing critical scientific and engineering models, such as the fractional Fisher equation [3], fractional stiff system [7], fractional Sharma-Tasso-Olever equation [17], fractional cancer tumor model [16], fractional fluids flow model[4], the fractional vibration model of large membranes [10], and fractional SIR Epidemic model [8]. Despite the fact that the FRPS method has been developed for various problems, there has not been much research on the fractional system of integro-differential equations.

This study aims to use the FRPS algorithm to solve a linear system of fractional Fredholm integro-differential equations (FIDEs),

$$
\begin{aligned}
& D^{\gamma_{i}} u_{i}(x)=g_{i}(x)+R_{i}\left(u_{1}(x), u_{2}(x), \ldots, u_{n}(x)\right) \\
& +\lambda_{i} \int_{a}^{b} \kappa_{i}(x, \tau) F_{i}\left(\tau, u_{1}(\tau), u_{2}(\tau), \ldots, u_{n}(\tau)\right) d \tau
\end{aligned}
$$

with initial condition $u_{i}(0)=c_{i, 0}, i=1,2,3, \ldots, n$, where $D^{\gamma_{i}}$ is fractional derivative operator in Caputo sense, $g_{i}(x)$ is a real function, $\kappa$ is kernel, $F_{i}, R_{i}$ are linear functions, $a, b, \lambda_{i}$ and $\gamma_{i}$ are constants, $0<\gamma_{i}<1$. This work is considered as an extension of [24].

The rest of the paper is managed as follows. Section 2 presents a basic definition of fractional integrals, fractional $\mathrm{Ca}-$ puto derivatives, and fractional power series. Section 3 describes the construction of fractional power series solutions for the system of fractional FIDEs. Illustrative examples are shown in the last section.

\section{The basic concept of fractional inte- gral, fractional derivative and frac- tional power series}

This section provides a fundamental idea of fractional calculus. The Riemann-Liouville fractional integral and the Caputo fractional derivative are presented. In addition, the primary notion and the facts related to fractional power series are mentioned.

Definition 2.1 [13] The Riemann-Liouville fractional integral operator of order $\gamma \geq 0$ is defined by

$$
J_{a}^{\gamma} \phi(x)= \begin{cases}\frac{1}{\Gamma(\gamma)} \int_{a}^{x} \frac{\phi(\tau)}{(x-\tau)^{1-\gamma}} d \tau, & \gamma>0, x>0 \\ \phi(x), & \gamma=0,\end{cases}
$$

where $\Gamma(\cdot)$ denotes the Gamma function.
Definition 2.2 [13] For $n-1<\gamma<n, n \in \mathbb{N}$. The Caputo fractional derivative operator of order $\gamma$ is defined by

$$
D_{a}^{\gamma} \phi(x)=\frac{1}{\Gamma(n-\gamma)} \int_{a}^{x}(x-\tau)^{-\gamma+n-1} \phi^{(n)}(\tau) d \tau, x>0,
$$

where the function $\phi(x)$ has absolutely continuous derivatives up to order $n-1$. Specially, if $\gamma=n \in \mathbb{N}, D_{a}^{\gamma} \phi(x)=\phi^{(n)}(x)$. In particular, if $0<\gamma<1$, we have

$$
D_{a}^{\gamma} \phi(x)=\frac{1}{\Gamma(1-\gamma)} \int_{a}^{x}(x-\tau)^{-\gamma} \phi^{\prime}(\tau) d \tau .
$$

The operators $D_{a}^{\gamma}$ and $J_{a}^{\gamma}$ satisfy the following properties:

1. $D_{a}^{\gamma} C=0$ for any constant $C \in \mathbb{R}$.

2. $D_{a}^{\gamma}(x-a)^{p}=\frac{\Gamma(p+1)}{\Gamma(p+1-\gamma)}(x-a)^{p-\gamma}$, for $n-1<\gamma<$ $n, p>n-1$, and it is equal to zero otherwise.

3. $J_{a}^{\gamma_{1}} J_{a}^{\gamma_{2}} \phi(x)=J_{a}^{\gamma_{2}} J_{a}^{\gamma_{1}} \phi(x)=J_{a}^{\gamma_{1}+\gamma_{2}} \phi(x)$.

4. $J_{a}^{\gamma} C=\frac{C}{\Gamma(\gamma+1)}(x-a)^{\gamma}$ for any constant $C \in \mathbb{R}$.

5. $J_{a}^{\gamma}(x-a)^{\mu}=\frac{\Gamma(\mu+1)}{\Gamma(\mu+\gamma+1)}(x-a)^{\gamma+\mu}, \mu>-1$.

6. $\left(J_{a}^{\gamma} D_{a}^{\gamma} \phi\right)(x)=\phi(x)-\sum_{k=0}^{\infty} \frac{\phi^{(k)}(a)}{k !}(x-a)^{k}$ for $\phi \in$ $C^{n}[a, b]$ and $n-1<\gamma<n$, with $n \in \mathbb{N}$. If $\gamma \geq 0, \phi \in$ $C[a, b]$, then $D^{\gamma} J^{\gamma} \phi(x)=\phi(x)$.

One can note that $D_{a}^{\gamma}$ and $J_{a}^{\gamma}$ are linear operators, that is for any constant $c_{1}, c_{2}$

$$
\begin{aligned}
D_{a}^{\gamma}\left(c_{1} \phi(x)+c_{2} \psi(x)\right) & =c_{1} D_{a}^{\gamma} \phi(x)+c_{2} D_{a}^{\gamma} \psi(x), \\
J_{a}^{\gamma}\left(c_{1} \phi(x)+c_{2} \psi(x)\right) & =c_{1} J_{a}^{\gamma} \phi(x)+c_{2} J_{a}^{\gamma} \psi(x) .
\end{aligned}
$$

Definition 2.3 [14] A power series expansion at $x=x_{0}$ of the following form

$$
\sum_{m=0}^{\infty} a_{m}\left(x-x_{0}\right)^{m \beta}=a_{0}+a_{1}\left(x-x_{0}\right)^{\beta}+a_{1}\left(x-x_{0}\right)^{2 \beta}+\ldots
$$

for $n-1<\beta \leq n, n \in \mathbb{N}$ and $x \leq x_{0}$, is called the fractional power series (FPS).

Theorem 2.1 [6] There are only three possibilities for the FPS $\sum_{m=0}^{\infty} a_{m}\left(x-x_{0}\right)^{m \beta}$, which are:

1. The series converges only for $x=x_{0}$. That is; the radius of convergence equals zero.

2. The series converges for all $x \geq x_{0}$. That is; the radius of convergence equals $\infty$.

3. The series converges for $x \in\left[x_{0}, x_{0}+R\right)$, for some positive real number $R$ and diverges for $x>x_{0}+R$. Here, $R$ is the radius of convergence for the FPS. 
The Fractional Residual Power Series Method for Solving a System of Linear Fractional Fredholm Integro-differential Equations

Theorem 2.2 [6] Suppose that $u(x)$ has a FPS representation at $x=x_{0}$ of the form

$$
u(x)=\sum_{m=0}^{\infty} c_{m}\left(x-x_{0}\right)^{m \beta}
$$

If $u(x) \in C\left[x_{0}, x_{0}+R\right)$, and $D^{m \beta} u(x) \in C\left[x_{0}, x_{0}+\right.$ $R)$, for $m=0,1,2, \ldots$, then the coefficients $c_{m}$ will be of the form $c_{m}=\frac{D^{m \beta} u\left(x_{0}\right)}{\Gamma(m \beta+1)}$, where $D^{m \beta}=D^{\beta} \cdot D^{\beta}$. $D^{\beta} \cdots D^{\beta}$ (mtimes $)$.

Theorem 2.3 [7] Let $u(x)$ has the FPS in (2) with radius of convergence $R>0$, and suppose that $u(x) \in C\left[x_{0}, x_{0}+\right.$ $R), D_{x_{0}}^{j \gamma} u(x) \in C\left(x_{0}, x_{0}+R\right)$ for $j=0,1,2, \ldots, N+1$. Then

$$
u(x)=u_{N}(x)+R_{N}(\xi)
$$

where $u_{N}(x)=\sum_{k=0}^{N} \frac{D_{x_{0}}^{k \gamma} u\left(x_{0}\right)}{\Gamma(k \gamma+1)}$ and $R_{N}(\xi)=$ $\frac{D_{x_{0}}^{(N+1) \gamma} u(\xi)}{\Gamma((N+1) \gamma+1)}\left(x-x_{0}\right)^{(N+1) \gamma}$, for some $\xi \in\left(x_{0}, x\right)$.

Theorem 2.4 [24] Let $\left|D_{x_{0}}^{(N+1) \gamma} u(x)\right| \leq K$, on $x_{0} \leq x<\xi$ for some constant $K$ where $N-1<\gamma \leq N$. Then, the reminder $R_{N}$ satisfies

$$
\left|R_{N}(x)\right| \leq \frac{K}{\Gamma((N+1) \gamma+1)}\left(x-x_{0}\right)^{(N+1) \gamma} .
$$

\section{Application of FRPS method to the system of fractional Fredholm integro-differential equations}

Assume that the FPS solution of the system (1) with initial conditions of $u_{i}(0)=c_{i}$ at $x=0$ has the following form:

$$
u_{i}(x)=\sum_{k=0}^{\infty} \frac{c_{i, k}}{\Gamma\left(1+k \gamma_{i}\right)} x^{k \gamma_{i}}, i=1, \ldots, n
$$

Using the initial condition, the solution can be written as

$$
u_{i}(x)=c_{i}+\sum_{k=1}^{\infty} \frac{c_{i, k}}{\Gamma\left(1+k \gamma_{i}\right)} x^{k \gamma_{i}}
$$

To follow the fractional power series method, let's suppose the approximate solution of the system (1)is in the form a $k$ thtruncated series:

$$
u_{i, k}(x)=c_{i}+\sum_{m=1}^{k} \frac{c_{i, m}}{\Gamma\left(1+m \gamma_{i}\right)} x^{m \gamma_{i}} .
$$

According to the RPS algorithm, the residual function is defined as

$\operatorname{Res} u_{i}(x)=D^{\gamma_{i}} u_{i}(x)-g_{i}(x)-R_{i}\left(u_{1}(x), u_{2}(x), \ldots, u_{n}(x)\right)$ $-\lambda_{i} \int_{a}^{b} \kappa_{i}(x, \tau) F_{i}\left(\tau, u_{1}(\tau), u_{2}(\tau), \ldots, u_{n}(\tau)\right) d \tau$, $i=1, \ldots, n, 0 \leq x<R$. Hence, the $k$ th-residual function $\operatorname{Res} u_{i, k}(x)$, for $k=1,2, \ldots, n$, are given by

$$
\begin{aligned}
& \operatorname{Res} u_{i, k}(x)=D^{\gamma_{i}} u_{i, k}(x)-g_{i}(x) \\
& -R_{i}\left(u_{1, k}(x), u_{2, k}(x), \ldots, u_{n, k}(x)\right) \\
& -\lambda_{i} \int_{a}^{b} \kappa_{i}(x, \tau) F_{i}\left(\tau, u_{1, k}(\tau), \ldots, u_{n, k}(\tau)\right) d \tau,
\end{aligned}
$$

As the results in [6], [14], [15], we have Res $u_{i}(x)=0$, and $\lim _{k \rightarrow \infty} \operatorname{Res} u_{i, k}(x)=\operatorname{Res} u_{i}(x)$, for any $x \geq 0$. These implies that $D^{m \gamma_{i}} \operatorname{Res} u_{i, k}(x)=0$ for $m=0,1,2, \ldots, k, i=$ $1,2, \ldots, n$, and $D^{n \gamma_{i}} \operatorname{Res} u_{i}(0)=D^{n \gamma_{i}} \operatorname{Res} u_{i, k}(0)=0$. Therefore, the coefficients of (3) can be found by solving the following equation:

$$
D^{(k-1) \gamma_{i}} \operatorname{Res} u_{i, k}(0)=0, i=1,2, \ldots, n, k=1,2, \ldots .
$$

To determine the coefficient $c_{i, 1}$ in (3), one substitutes the 1-st residual power series approximate solution,

$$
u_{i, 1}(x)=c_{i}+c_{i, 1} \frac{x^{\gamma_{i}}}{\Gamma\left(1+\gamma_{i}\right)}, \quad i=1,2, \ldots, n,
$$

into equation (4) with $k=1$, to obtain

$$
\begin{aligned}
\operatorname{Res} u_{i, 1}(x)= & D^{\gamma_{i}} u_{i, 1}(x)-g_{i}(x)-R_{i}\left(u_{1,1}(x), \ldots, u_{n, 1}(x)\right) \\
- & \lambda_{i} \int_{a}^{b} \kappa_{i}(x, \tau) F_{i}\left(\tau, u_{1,1}(\tau), \ldots, u_{n, 1}(\tau)\right) d \tau \\
= & D^{\gamma_{i}}\left(c_{i}+c_{i, 1} \frac{x^{\gamma_{i}}}{\Gamma\left(1+\gamma_{i}\right)}\right)-g_{i}(x) \\
- & R_{i}\left(c_{1}+c_{1,1} \frac{x^{\gamma_{1}}}{\Gamma\left(1+\gamma_{1}\right)}, c_{2}+c_{2,1} \frac{x^{\gamma_{2}}}{\Gamma\left(1+\gamma_{2}\right)},\right. \\
& \left.\quad \ldots, c_{n}+c_{n, 1} \frac{x^{\gamma_{n}}}{\Gamma\left(1+\gamma_{n}\right)}\right) \\
- & \lambda_{i} \int_{a}^{b} \kappa_{i}(x, \tau) F_{i}\left(c_{1}+c_{1,1} \frac{\tau^{\gamma_{1}}}{\Gamma\left(1+\gamma_{1}\right)},\right. \\
& \left.\quad \ldots, c_{n}+c_{n, 1} \frac{\tau^{\gamma_{n}}}{\Gamma\left(1+\gamma_{n}\right)}\right) d \tau .
\end{aligned}
$$

Using the fact that $\operatorname{Res} u_{i, 1}(0)=0, i=1,2, \ldots, n$, we have a linear system of $n$ equations with $n$ unknowns,

$$
\begin{array}{r}
c_{i, 1}-g_{i}(0)-R_{i}\left(c_{1}, c_{2}, \ldots, c_{n}\right) \\
=\lambda_{i} \int_{a}^{b} \kappa_{i}(0, \tau) F_{i}\left(c_{1}+c_{1,1} \frac{\tau^{\gamma_{1}}}{\Gamma\left(1+\gamma_{1}\right)},\right. \\
\left.\ldots, c_{n}+c_{n, 1} \frac{\tau^{\gamma_{n}}}{\Gamma\left(1+\gamma_{n}\right)}\right) d \tau,
\end{array}
$$

which can be solved for $c_{i, 1}, i=1,2, \ldots, n$. Substituting these coefficients into [6], one obtains the 1st-truncated series solution of (1). In a similar way, to find the coefficients $c_{i, 2}, i=1,2, \ldots, n$, we can substitute

$$
u_{i, 2}(x)=c_{i}+c_{i, 1} \frac{x^{\gamma_{i}}}{\Gamma\left(1+\gamma_{i}\right)}+c_{i, 2} \frac{x^{2 \gamma_{i}}}{\Gamma\left(1+2 \gamma_{i}\right)}
$$

into equation (4) with $k=2$. Using the condition $D^{\gamma_{i}} \operatorname{Res} u_{i, 2}(0)=0$, yields a system of linear equations that can be solved for $c_{i, 2}, i=1,2, \ldots, n$. By (7), the 2 ndtruncated power series solution of (1) is proposed. By repeating the same routine until arbitrary order is obtained, the other unknown coefficient, $c_{i, k}$ will be determined [1], [9]. 


\section{Illustrative Examples}

In this section, we verify the efficiency and accuracy of the proposed method by employing them to solve some fractional systems of linear FIDEs with a known solution. Some derived steps are simplified via the properties of the Gamma function.

Example 4.1 Consider the following fractional system of linear Fredholm integro-differential equations

$$
\begin{aligned}
& D^{\frac{1}{2}} u(x)=g_{1}(x)+3 u-v-\int_{0}^{1} x t^{\frac{1}{2}}(u(t)-2 v(t)) d t \\
& D^{\frac{1}{2}} v(x)=g_{2}(x)-5 u+2 v-\int_{0}^{1} x^{2} t(2 u(t)+v(t)) d t
\end{aligned}
$$

subject to initial conditions

$$
u(0)=2, v(0)=1,
$$

where $g_{1}(x)=\frac{\sqrt{\pi}}{2}-5-3 x^{\frac{1}{2}}+2 x^{\frac{3}{2}}-\frac{5}{6} x, g_{2}(x)=8+5 x^{\frac{1}{2}}+$ $\frac{3 \sqrt{\pi}}{2} x-4 x^{\frac{3}{2}}+\frac{271}{70} x^{2}$. The exact solution of this problem is $u(x)=2+x^{\frac{1}{2}}, v(x)=1+2 x^{\frac{3}{2}}$.

Here, we have $\gamma_{1}=\gamma_{2}=\frac{1}{2}, c_{1}=2, c_{2}=1$, and by utilizing the initial conditions, the $k$ th-truncated FPS approximate solution to this problem is written as

$u_{k}(x)=2+\sum_{n=1}^{k} \frac{a_{n}}{\Gamma\left(\frac{n}{2}+1\right)} x^{\frac{n}{2}}, v_{k}(x)=1+\sum_{n=1}^{k} \frac{b_{n}}{\Gamma\left(\frac{n}{2}+1\right)} x^{\frac{n}{2}}$

For $k=1$, suppose the 1st-truncated FPS approximate solution is

$$
u_{1}(x)=2+\frac{a_{1}}{\Gamma\left(\frac{3}{2}\right)} x^{\frac{1}{2}}, v_{1}(x)=1+\frac{b_{1}}{\Gamma\left(\frac{3}{2}\right)} x^{\frac{1}{2}} .
$$

Substituting (10) into (8)-(9), the 1st-residual functions are

$$
\begin{aligned}
\operatorname{Res} u_{1}(x)= & a_{1}-\frac{\sqrt{\pi}}{2}+\left(3+\frac{2 b_{1}-6 a_{1}}{\sqrt{\pi}}\right) x^{\frac{1}{2}} \\
& +\left(\frac{5}{6}+\frac{a_{1}-2 b_{1}}{\sqrt{\pi}}\right) x-2 x^{\frac{3}{2}}, \\
\operatorname{Res} v_{1}(x)= & b_{1}+\left(\frac{10 a_{1}-4 b_{1}}{\sqrt{\pi}}-5\right) x^{\frac{1}{2}}-\frac{3 \sqrt{\pi}}{2} x+4 x^{\frac{3}{2}} \\
& +\left(\frac{8 a_{1}+4 b_{1}}{5 \sqrt{\pi}}-\frac{48}{35}\right) x^{2} .
\end{aligned}
$$

From [5], $\operatorname{Res} u_{1}(0)=0, \operatorname{Res} v_{1}(0)=0$, yields $a_{1}=$ $\frac{\sqrt{\pi}}{2}, b_{1}=0$. Thus, the 1st-truncated FPS approximate solution is $u_{1}(x)=2+x^{\frac{1}{2}}, v_{1}(x)=1$. Next, consider $k=2$, the 2nd-truncated FPS approximation is expressed in the form

$$
u_{2}(x)=2+x^{\frac{1}{2}}+a_{2} x, v_{2}(x)=1+b_{2} x .
$$

After substituting (11) into (8)-(9), one gets the 2nd-residual functions

$$
\begin{aligned}
\operatorname{Res} u_{2}(x)= & \frac{2 a_{2}}{\sqrt{\pi}} x^{\frac{1}{2}}+\left(\frac{4}{3}+\frac{b_{2}-13 a_{2}}{5}\right) x-2 x^{\frac{3}{2}} \\
\operatorname{Res} v_{2}(x)= & \frac{2 b_{2}}{\sqrt{\pi}} x^{\frac{1}{2}}+\left(5 a_{2}-2 b_{2}-\frac{3 \sqrt{\pi}}{2}\right) x+4 x^{\frac{3}{2}} \\
& +\left(\frac{2 a_{2}+b_{2}}{3}-\frac{4}{7}\right) x^{2}
\end{aligned}
$$

Then, employing the conditions $D^{\frac{1}{2}} \operatorname{Res} u_{2}(0)=0$, $D^{\frac{1}{2}} \operatorname{Res} v_{2}(0)=0$, we reach the coefficients of 11 , $a_{2}=0$, $b_{2}=0$. So, the 2nd-truncated FPS approximate solution is $u_{2}(x)=2+x^{\frac{1}{2}}, v_{2}(x)=1$.

For $k=3$, assume the 3rd-truncated FPS approximate solution as

$$
u_{3}(x)=2+x^{\frac{1}{2}}+\frac{a_{3}}{\Gamma\left(\frac{5}{2}\right)} x^{\frac{3}{2}}, v_{3}(x)=1+\frac{b_{3}}{\Gamma\left(\frac{5}{2}\right)} x^{\frac{3}{2}} .
$$

Substituting (12) into (8)-(9), the 3rd residual functions are found

$$
\begin{aligned}
\operatorname{Res} u_{3}(x)= & \left(a_{3}+\frac{4}{3}+\frac{4 a_{3}-8 b_{3}}{9 \sqrt{\pi}}\right) x+\left(\frac{4 b_{3}-4 a_{3}}{3 \sqrt{\pi}}-2\right) x^{\frac{3}{2}}, \\
\operatorname{Res} v_{3}(x)= & \left(b_{3}-\frac{3 \sqrt{\pi}}{2}\right) x+\left(4+\frac{20 a_{3}-8 b_{3}}{3 \sqrt{\pi}}\right) x^{\frac{3}{2}} \\
& +\left(\frac{16 a_{3}+8 b_{3}}{21 \sqrt{\pi}}-\frac{4}{7}\right) x^{2} .
\end{aligned}
$$

Applying the conditions (5), $\frac{d}{d x} \operatorname{Res} u_{3}(0)=0$, $\frac{d}{d x} \operatorname{Res} v_{3}(0)=0$, leads to $a_{3}=0, b_{3}=\frac{3 \sqrt{\pi}}{2}$. Thus, the 3rdtruncated FPS approximation is $u_{3}(x)=2+x^{\frac{1}{2}}, v_{3}(x)=$ $1+2 x^{\frac{3}{2}}$.

Repeating the same procedure for $k \geq 4$, one finds that the coefficients of the FPS approximate solution are $a_{k}=0$, $b_{k}=0$ for $k \geq 4$. Therefore, the obtained FPS solution to this problem is

$$
u(x)=2+x^{\frac{1}{2}}, v(x)=1+2 x^{\frac{3}{2}} .
$$

Example 4.2 Now we consider the linear system of fractional FIDEs

$$
\begin{aligned}
& D^{\frac{1}{4}} u(x)=g_{1}(x)+3 u+2 v-\frac{8}{243} \int_{0}^{1} x^{2} t^{\frac{2}{3}}(2 u(t)+3 v(t)) d t, \\
& D^{\frac{1}{3}} v(x)=g_{2}(x)-6 u-4 v+66 \int_{0}^{1} x t(u(t)-5 v(t)) d t
\end{aligned}
$$

where $g_{1}(x)=\frac{3 \Gamma\left(\frac{3}{4}\right)}{2 \sqrt{\pi}} x^{\frac{1}{2}}+3 x^{\frac{3}{4}}+2 x+\frac{1}{29} x^{2}, g_{2}(x)=$ $\frac{3}{2 \Gamma\left(\frac{2}{3}\right)} x^{\frac{2}{4}}-6 x^{\frac{3}{4}}+471 x$ with initial conditions

$$
u(0)=2, v(0)=-3 \text {. }
$$

The exact solution is $u(x)=x^{\frac{3}{4}}+2, v(x)=x-3$.

Since $\gamma_{1}=\frac{1}{4}, \gamma_{2}=\frac{1}{3}$ and $c_{1}=2, c_{2}=-3$, the $k$ th-truncated FPS approximate solution to the problem is of the form

$u_{k}(x)=2+\sum_{n=1}^{k} \frac{a_{n}}{\Gamma\left(\frac{n}{4}+1\right)} x^{\frac{n}{4}}, v_{k}(x)=-3+\sum_{n=1}^{k} \frac{b_{n}}{\Gamma\left(\frac{n}{3}+1\right)} x^{\frac{n}{3}}$.

For $k=1$, one finds that the 1st-truncated FPS approximate solution is

$$
u_{1}(x)=2+\frac{a_{1}}{\Gamma\left(\frac{5}{4}\right)} x^{\frac{1}{4}}, v_{1}(x)=-3+\frac{b_{1}}{\Gamma\left(\frac{4}{3}\right)} x^{\frac{1}{3}} .
$$


The Fractional Residual Power Series Method for Solving a System of Linear Fractional Fredholm Integro-differential Equations

We substitute (15) into (13)-(14), then the 1st-residual functions are observed

$$
\begin{aligned}
\operatorname{Res} u_{1}(x)= & a_{1}+\frac{6 \sqrt{2} \Gamma\left(\frac{3}{4}\right) a_{1}}{\pi} x^{\frac{1}{4}}+\frac{3 \sqrt{3} \Gamma\left(\frac{2}{3}\right) b_{1}}{\pi} x^{\frac{1}{3}} \\
& -\frac{3 \Gamma\left(\frac{3}{4}\right)}{2 \sqrt{\pi}} x^{\frac{1}{2}}-3 x^{\frac{3}{4}}-2 x \\
& +\left(\frac{151}{2349}-\frac{128 \sqrt{2} \Gamma\left(\frac{3}{4}\right) a_{1}}{1863 \pi}-\frac{2 \sqrt{3} \Gamma\left(\frac{2}{3}\right) b_{1}}{27 \pi}\right) x^{2}, \\
\operatorname{Res} v_{1}(x)= & b_{1}-\frac{12 \sqrt{2} \Gamma\left(\frac{3}{4}\right) a_{1}}{\pi} x^{\frac{1}{4}}-\frac{6 \sqrt{3} \Gamma\left(\frac{2}{3}\right) b_{1}}{\pi} x^{\frac{1}{3}} \\
& -\frac{3}{2 \Gamma\left(\frac{2}{3}\right)} x^{\frac{2}{3}}+6 x^{\frac{3}{4}} \\
& +\left(\frac{176 \sqrt{2} \Gamma\left(\frac{3}{4}\right) a_{1}}{3 \pi}-\frac{1485 \sqrt{3} \Gamma\left(\frac{2}{3}\right) b_{1}}{7 \pi}+90\right) x .
\end{aligned}
$$

By the condition (5), it deduces that Res $u_{1}(0)=0$, Res $v_{1}(0)=0$ and conducts to $a_{1}=0, b_{1}=0$. For $k=2$, we can write the 2nd-truncated FPS approximation in the form

$$
u_{2}(x)=2+\frac{a_{2}}{\Gamma\left(\frac{3}{2}\right)} x^{\frac{1}{2}}, v_{2}(x)=-3+\frac{b_{2}}{\Gamma\left(\frac{5}{3}\right)} x^{\frac{2}{3}}
$$

and the 2nd-residual functions are obtained

$$
\begin{aligned}
\operatorname{Res} u_{2}(x)= & \frac{2 \sqrt{2} \Gamma\left(\frac{3}{4}\right) a_{2}}{\pi} x^{\frac{1}{4}}+\left(\frac{6 a_{2}}{\sqrt{\pi}}-\frac{3 \Gamma\left(\frac{3}{4}\right)}{2 \sqrt{\pi}}\right) x^{\frac{1}{2}} \\
& +\frac{3 b_{2}}{\Gamma\left(\frac{2}{3}\right)} x^{\frac{2}{3}}-3 x^{\frac{3}{4}}-2 x \\
& +\left(\frac{151}{2349}-\frac{64 a_{2}}{1053 \sqrt{2}}-\frac{4 b_{2}}{63 \Gamma\left(\frac{2}{3}\right)}\right) x^{2} \\
\operatorname{Res} v_{2}(x)= & \frac{3 \sqrt{3} \Gamma\left(\frac{2}{3}\right) b_{2}}{2 \pi} x^{\frac{1}{3}}-\frac{12 a_{2}}{\sqrt{\pi}} x^{\frac{1}{2}} \\
& -\left(\frac{6 b_{2}}{\Gamma\left(\frac{2}{3}\right)}+\frac{3}{2 \Gamma\left(\frac{2}{3}\right)}\right) x^{\frac{2}{3}} \\
& +6 x^{\frac{3}{4}}+\left(\frac{264 a_{2}}{5 \sqrt{\pi}}-\frac{1485 b_{2}}{8 \Gamma\left(\frac{2}{3}\right)}+90\right) x .
\end{aligned}
$$

Apply the conditions, $D^{\frac{1}{4}} \operatorname{Res} u_{2}(0)=0$, and $D^{\frac{1}{3}} \operatorname{Res} v_{2}(0)=$ 0 , the unknown coefficients are found, $a_{2}=0, b_{2}=0$. Next, suppose the 3rd-truncated FPS approximate solution in the form

$$
u_{3}(x)=2+\frac{a_{3}}{\Gamma\left(\frac{7}{4}\right)} x^{\frac{3}{4}}, v_{3}(x)=-3+\frac{b_{3}}{\Gamma(2)} x .
$$

Substituting (16) into (13)-(14), we get the 3rd-residual functions of the problem

$$
\begin{aligned}
\operatorname{Res} u_{3}(x)= & \left(\frac{2 a_{3}}{\sqrt{\pi}}-\frac{3 \Gamma\left(\frac{3}{4}\right)}{2 \sqrt{\pi}}\right) x^{\frac{1}{2}}+\left(\frac{4 a_{3}}{\Gamma\left(\frac{3}{4}\right)}-3\right) x^{\frac{3}{4}} \\
& +2\left(b_{3}-1\right) x+\left(\frac{151}{2349}-\frac{256 a_{3}}{7047 \Gamma\left(\frac{3}{4}\right)}-\frac{b_{3}}{27}\right) x^{2},
\end{aligned}
$$$$
\operatorname{Res} v_{3}(x)=\frac{3\left(b_{3}-1\right)}{2 \Gamma\left(\frac{2}{3}\right)} x^{\frac{2}{3}}+\left(6-\frac{8 a_{3}}{\Gamma\left(\frac{3}{4}\right)}\right) x^{\frac{3}{4}}
$$$$
+\left(90-114 b_{3}\right) x \text {. }
$$

After using the conditions $D^{\frac{1}{2}} \operatorname{Res} u_{3}(0)=0$, and $D^{\frac{2}{3}} \operatorname{Res}$ $v_{2}(0)=0$, one finds that $a_{3}=\frac{3}{4} \Gamma\left(\frac{3}{4}\right), b_{3}=1$. Replicating the above technique for $k \geq 4$, the coefficients of FPS approximation are determined, $a_{k}=0, b_{k}=0, k \geq 4$. Therefore, the FPS approximate solution to this problems is $u(x)=x^{\frac{3}{4}}+2, v(x)=x-3$ which is the exact.

Example 4.3 Next, we consider the system of linear fractional FIDEs

$$
\begin{aligned}
& D^{\frac{2}{3}} u(x)=g_{1}(x)+u+3 v-\frac{1}{4} \int_{0}^{1} x t^{-\frac{1}{2}}(3 u(t)+4 v(t)) d t, \\
& D^{\frac{3}{4}} v(x)=g_{2}(x)-3 u-9 v-\int_{0}^{1} x^{2} t(2 u(t)-v(t)) d t
\end{aligned}
$$

where $g_{1}(x)=-\frac{2^{\frac{4}{3}} \sqrt{\pi}}{3 \Gamma\left(\frac{5}{6}\right)} x^{\frac{2}{3}}+\frac{57}{22} x+x^{\frac{4}{3}}-3 x^{\frac{3}{2}}, g_{2}(x)=$ $\frac{\sqrt{\pi}}{\Gamma\left(\frac{3}{4}\right)} x^{\frac{3}{4}}-3 x^{\frac{4}{3}}+9 x^{\frac{3}{2}}+\frac{183}{70} x^{2}$ with initial conditions

$$
u(0)=3, v(0)=-1 \text {. }
$$

The exact solution is $u(x)=3-x^{\frac{4}{3}}, v(x)=-1+x^{\frac{3}{2}}$.

Note that $\gamma_{1}=\frac{2}{3}, \gamma_{2}=\frac{3}{4}$ and the initial condition gives $c_{1}=$ $3, c_{2}=-1$. The $k$ th-truncated FPS approximate solution of the problem is

$$
\begin{gathered}
u_{k}(x)=3+\sum_{n=1}^{k} \frac{a_{n}}{\Gamma\left(\frac{2 n}{3}+1\right)} x^{\frac{2 n}{3}}, \\
v_{k}(x)=-1+\sum_{n=1}^{k} \frac{b_{n}}{\Gamma\left(\frac{3 n}{4}+1\right)} x^{\frac{3 n}{4}} .
\end{gathered}
$$

For $k=1$, suppose that the 1st-truncated FPS approximate solution is

$$
u_{1}(x)=3+\frac{a_{1}}{\Gamma\left(\frac{5}{3}\right)} x^{\frac{2}{3}}, v_{1}(x)=-1+\frac{b_{1}}{\Gamma\left(\frac{7}{4}\right)} x^{\frac{3}{4}} .
$$

We substitute (19) into (17)-(18), then the 1st- residual functions are written as

$$
\begin{aligned}
\operatorname{Res} u_{1}(x)= & a_{1}+\left(\frac{2^{\frac{4}{3}} \sqrt{\pi}}{3 \Gamma\left(\frac{5}{3}\right)}-\frac{3 a_{1}}{2 \Gamma\left(\frac{2}{3}\right)}\right) x^{\frac{2}{3}}-\frac{4 b_{1}}{\Gamma\left(\frac{3}{4}\right)} x^{\frac{3}{4}} \\
& +\left(\frac{27 a_{1}}{28 \Gamma\left(\frac{2}{3}\right)}+\frac{16 b_{1}}{15 \Gamma\left(\frac{3}{4}\right)}-\frac{1}{11}\right) x-x^{\frac{4}{3}}+3 x^{\frac{3}{2}}, \\
\operatorname{Res} v_{1}(x)= & b_{1}+\left(\frac{12 b_{1}-\sqrt{\pi}}{\Gamma\left(\frac{3}{4}\right)}\right) x^{\frac{3}{4}}+\frac{9 a_{1}}{2 \Gamma\left(\frac{2}{3}\right)} x^{\frac{2}{3}}+3 x^{\frac{4}{3}} \\
& -9 x^{\frac{3}{2}}+\left(\frac{31}{35}-\frac{16 b_{1}}{33 \Gamma\left(\frac{3}{4}\right)}+\frac{9 a_{1}}{8 \Gamma\left(\frac{2}{3}\right)}\right) x^{2} .
\end{aligned}
$$

Following the condition $\operatorname{Res} u_{1}(0)=0, \operatorname{Res} v_{1}(0)=0$, we find that the coefficients of FPS $(19)$ are $a_{1}=0, b_{1}=0$. So, the 1st-truncated FPS approximate solution is $u_{1}(x)=3, v_{1}(x)=$ -1 . For $k=2$, the 2nd-truncated FPS approximation is mentioned

$$
u_{2}(x)=3+\frac{a_{2}}{\Gamma\left(\frac{4}{3}+1\right)} x^{\frac{4}{3}}, v_{2}(x)=-1+\frac{b_{2}}{\Gamma\left(\frac{3}{2}+1\right)} x^{\frac{3}{2}} .
$$


Substituting (20) into [17)-(18), one gets the 2nd residual functions

$$
\begin{aligned}
\operatorname{Res} u_{2}(x)= & \left(\frac{3 a_{2}}{2 \Gamma\left(\frac{2}{3}\right)}+\frac{2^{\frac{4}{3}} \sqrt{\pi}}{3 \Gamma\left(\frac{5}{6}\right)}\right) x^{\frac{2}{3}} \\
& +\left(\frac{81 \sqrt{3} \Gamma\left(\frac{2}{3}\right) a_{2}}{176 \pi}+\frac{2 b_{2}}{3 \sqrt{\pi}}-\frac{1}{11}\right) x \\
& -\left(1+\frac{9 \sqrt{3} \Gamma\left(\frac{2}{3}\right) a_{2}}{8 \pi}\right) x^{\frac{4}{3}}+\left(3-\frac{4 b_{2}}{\sqrt{\pi}}\right) x^{\frac{3}{2}}, \\
\operatorname{Res} v_{2}(x)= & \left(\frac{4 b_{2}}{3 \Gamma\left(\frac{3}{4}\right)}-\frac{\sqrt{\pi}}{\Gamma\left(\frac{3}{4}\right)}\right) x^{\frac{3}{4}}+\left(3+\frac{27 \sqrt{3} \Gamma\left(\frac{2}{3}\right) a_{2}}{8 \pi}\right) x^{\frac{4}{3}} \\
& +\left(\frac{27 \sqrt{3} \Gamma\left(\frac{2}{3}\right) a_{2}}{40 \pi}-\frac{8 b_{2}}{21 \sqrt{\pi}}+\frac{31}{35}\right) x^{2} \\
& +\left(\frac{12 b_{2}}{\sqrt{\pi}}-9\right) x^{\frac{3}{2}} .
\end{aligned}
$$

From the condition 5 , we deduce that $D^{\frac{2}{3}} \operatorname{Res} u_{2}(0)=0$, $D^{\frac{3}{4}} \operatorname{Res} v_{2}(0)=0$, and thus $a_{2}=-\frac{8 \sqrt{3} \pi}{27 \Gamma\left(\frac{2}{3}\right)}, b_{2}=\frac{3 \sqrt{\pi}}{4}$. Therefore, the 2nd-truncated FPS approximate solution is $u_{2}(x)=$ $3-x^{\frac{4}{3}}, v_{2}(x)=-1+x^{\frac{3}{2}}$. After repeating the same routine for $k \geq 3$, one gets that $a_{k}=0, b_{k}=0$ and $u_{k}(x)=3-x^{\frac{4}{3}}$, $v_{k}(x)=-1+x^{\frac{3}{2}}$ for $k \geq 3$. Thus, the FPS solution to this problem is

$$
u(x)=3-x^{\frac{4}{3}}, v(x)=-1+x^{\frac{3}{2}} .
$$

\section{Conclusions}

For applying the FRPS method to the linear system of FIDEs, we start by assuming the approximate solution as a truncated fractional power series that satisfies the initial condition. Later, define a residual function by substituting the truncated fractional power series into the original system. An easily solved linear system of an algebraic equation is addressed by requiring conditions (5). One can note that solving the linear algebraic system may not need a standard method. Each equation can be solved separately. Finally, the unknown coefficients of the fractional series solution are determined and the approximate fractional power series solution is obtained. As demonstrated in the preceding examples, when the exact solution is a fractional polynomial function of degree up to $k \gamma$, the derived $k \gamma$ approximate power series is exact.

In conclusion, the FRPS method is an analytical, powerful method for constructing the fractional power series solution of the linear system of FIDEs. When compared to the procedure in [23], [22] the proposed method is simpler, more convenient, and more accurate. Testing examples with known solutions demonstrate the efficiency and accuracy of this technique.

\section{Acknowledgements}

This research project was financially supported by Mahasarakham University 2021. The author is deeply indebted to the reviewers for their valuable comments and suggestions. Thanks to Ms. Phakakong Temchit and Mr. Chonlata Chaikhiew for their frequent help.

\section{REFERENCES}

[1] Akgül A., Inc M., Karatas E. and Baleanu D., "Numerical solutions of fractional differential equations of Lane - Emden type by an accurate technique," Advances in Difference Equations, vol. 2015, 220, 2015. DOI: 10.1186/s13662-015-0558-8.

[2] Al-Marashi Adel A., "Approximate solution of the system of linear fractional integro-differential equations of Volterra using B-spline method," American Review of Mathematics and Statistics, vol. 3, no. 2, pp. 39-47, 2015. DOI: 10.15640/arms.v3n2a6.

[3] Al Qurashi1 M. M., Korpinar Z., Baleanu D., Inc M., "A new iterative algorithm on the time-fractional Fisher equation: Residual power series method," Advances in Mechanical Engineering, vol.9, no. 9, pp. 1-8, 2017. DOI: $10.1177 / 1687814017716009$.

[4] Arafa A. and Elmahdy G.,"Application of Residual Power Series Method to Fractional Coupled Physical Equations Arising in Fluids Flow," International Journal of Differential Equations, vol. 2018, Article ID 7692849, 10 pages, 2018. DOI: $10.1155 / 2018 / 7692849$.

[5] Didgar M., Vahidi A.R. and Biazar J.,"An approximate approach for system of fractional integro-differential equations based on Taylor expansion," Kragujevac Journal of Mathematics, vol. 44, no. 3, pp. 379-392, 2020. DOI 10.46793/KgJMat2003.379D.

[6] El-Ajou A., Arqub O. A.and Al-Smadi M., "A general form of the generalized Taylor's formula with some applications," Applied Mathematics and Computation, vol. 256, pp. 851-859, 2015. DOI: 10.1016/j.amc.2015.01.034.

[7] Freihet A. et el., "Construction of fractional power series solutions to fractional stiff system using residual functions algorithm," Advances in Difference Equations, vol. 2019, 95, 2019. DOI: 10.1186/s13662-019-2042-3.

[8] Hasan Sh. et el.,"Solution of Fractional SIR Epidemic Model using Residual Power Series Method," Applied Mathematics \& Information Sciences, vol.13, no.2, pp. 153-161, 2019.DOI: 10.18576/amis/130202.

[9] Inc M., Korpinar Z. Al Qurashi M.M. and Baleanu D.,"A new method for approximate solutions of some nonlinear equations: Residual power series method," Advances in Mechanical Engineering, vol. 8, no. 4, pp. 1-7, 2016. DOI: $10.1177 / 1687814016644580$.

[10] Jena R. M. and Chakraverty S., "Residual Power Series Method for Solving Time-fractional Model of Vibration Equation of Large Membranes," Journal of Applied and Computational Mechanics, vol.5, no. 4, pp. 603-615, 2019. DOI: 10.22055/JACM.2018.26668.1347. 
The Fractional Residual Power Series Method for Solving a System of Linear Fractional Fredholm Integro-differential 798 Equations

[11] Khader M. M. and Sweilam N.H.,"On the approximate solutions for system of fractional integro-differential equations using Chebyshev pseudo-spectral method," Applied Mathematical Modelling, vol. 37, no. 24, pp. 9819-9828, 2013. DOI: 10.1016/j.apm.2013.06.010.

[12] Khan H., Arif M., Mohyud-Din S. T., Bushnaq S.,"Numerical solutions to systems of fractional Voltera Integro differential equations, using Chebyshev wavelet method," Journal of Taibah University for Science, vol. 12, no. 5, pp. 584-591, 2018. DOI: 10.1080/16583655.2018.1510149.

[13] Kilbas A., Srivastava H. and Trujillo J., Theory and Applications of Fractional Differential Equations, 1st ed. Elsevier, 2006.

[14] Komashynska I., Al-Smadi M., Arqub O. A. and Momani Sh., "An efficient analytical method for solving singular initial value problems of nonlinear systems," Applied Mathematics \& Information Sciences, vol.10, no. 2, pp. 647-656, 2016. DOI: 10.18576/amis/100224.

[15] Komashynska I., Al-Smadi M., Ateiwi A. and Al-Obaidy S.,"Approximate analytical solution by residual power series method for system of Fredholm integral equations," Applied Mathematics \& Information Sciences, vol.10, no. 3, pp. 975985, 2016. DOI: 10.18576/amis/100315.

[16] Korpinar Z., Inc M., Hınçal E. and Baleanu D.,"Residual power series algorithm for fractional cancer tumor models," Alexandria Engineering Journal, vol. 59, no. 3, pp. 1405-1412, 2020. DOI: 10.1016/j.aej.2020.03.044.

[17] Kumar A., Kumar S. and Singh M., "Residual power series method for fractional Sharma-Tasso-Olever equation," Communications in Numerical Analysis, vol. 2016, no. 1, pp. 1-10, 2016. DOI: 10.5899/2016/CNA-00235.

[18] Loh J.R.and Phang C., "A new numerical scheme for solving system of Volterra integro-differential equation,"Alexandria Engineering Journal, vol. 57, no. 2, pp. 1117-1124, 2018. DOI: 10.1016/j.aej.2017.01.021.

[19] Momani S. and Qaralleh R., "An Efficient Method for Solving Systems of Fractional Integro-Differential Equations," Comput- ers \& Mathematics with Applications, vol. 52, no. 3-4, pp. 459470, 2006. DOI: 10.1016/j.camwa.2006.02.011.

[20] Oliveira E. C. and Machado J. A. T., "A Review of Definitions for Fractional Derivatives and Integral, Mathematical Problems in Engineering," vol. 2014, Art. ID 238459, 6 pages, 2014. DOI: $10.1155 / 2014 / 238459$.

[21] Pue-on P., "The modified Sadik decomposition method to solve a system of nonlinear fractional Volterra integro-differential equations of convolution type," WSEAS Transactions on Mathematics, vol. 20, no. 34, pp. 335-343, 2021. DOI: 10.37394/23206.2021.20.34.

[22] Saeed R. K. and Sdeq H. M.,"Solving a System of Linear Fredholm Fractional Integro-differential Equations Using Homotopy Perturbation Method." Australian Journal of Basic and Applied Sciences, vol.4, no. 4, pp. 633-638, 2010. http://www. ajbasweb.com/old/ajbas_april_2010. html

[23] Saleh M. H., Mohamed D.Sh., Ahmed M.H. and Marjan M.K., "System of linear fractional integro-differential equations by using Adomian decomposition method," International Journal of Computer Applications, vol. 121, no. 24, pp. 9-19, 2015. DOI: 10.5120/21872-5117.

[24] Syam M. I., "Analytical Solution of the Fractional Fredholm Integrodifferential Equation Using the Fractional Residual Power Series Method," Complexity, vol. 2017, Article ID 4573589, 6 pages, 2017. DOI: 10.1155/2017/4573589.

[25] Zedan H. A., Tantawy S. Sh. and Sayed Y. M., "New solutions for system of fractional integro-differential equations and Abel's integral equations by Chebyshev spectral method," Mathematical Problems in Engineering, vol. 2017, Article ID 7853839. 13 pages, 2017. DOI: 10.1155/2017/7853839.

[26] Zurigat M., Momani Sh. and Alawneh A., "Homotopy Analysis Method for Systems of Fractional Integro-Differential Equations," Neural, Parallel \& Scientific Computations, vol. 17, no. 2, pp. 169-189, 2009. http://dl.acm.org/citation. cfm?id=1737808. 